\title{
Role of Uroflowmetry in Children with Ano-Rectal Malformation in Anticipating Upper Urinary Tract Damage
}

\author{
Rahul Saxena ${ }^{\prime}$, Arvind Sinha ${ }^{\prime}$, Manish Pathak', Avinash S Jadhav ${ }^{\prime}$, Ankur Bansal ${ }^{2}$ \\ All India Institute of Medical Sciences, Jodhpur', \\ SR Medical Institute and Research Centre, Agra ${ }^{2}$.
}

\begin{abstract}
Background: The lower urinary tract dysfunction (LUTD) has high incidence in children with anorectal malformation (ARM) which if left untreated leads to upper tract damage.
\end{abstract}

Aim: To determine role of uroflowmetry in early diagnosis of LUTD in children with ARM.

Methods: This prospective study included twenty consecutive patients of ARM and every patient underwent uroflowmetry at-least 6 weeks after definitive procedure.

Results: The mean age of patients was 3.015 \pm 0.86 years. Of the twenty patients, there were $12(60 \%)$ males and $8(40 \%)$ females; 11 (55\%) were high ARM, $4(20 \%)$ were intermediate and 5(25\%) were low ARM. Lower urinary tract symptoms (LUTS) was present in $8 / 20$ (40\%) patients but uroflowmetric abnormalities were present in $11 / 20(55 \%)$ patients. Forty five percent $(5 / 11)$ patients with abnormal uroflowmetry were asymptomatic and 25\% (2/8) symptomatic patients had normal uroflowmetry. The incidence of uroflowmetric abnormalities was significantly higher in patients with spinal anomalies $\left(\mathrm{p}=0.03 ; \chi^{2}=4.1\right)$ and those with high $\mathrm{ARM}\left(\mathrm{p}=0.004 ; \chi^{2}=8.1\right)$.

Conclusion: Uroflowmetry is a noninvasive method that may help in early detection of neurovesical dysfunction in asymptomatic children and subsequent cystometric analysis in patients with uroflowmetric abnormalities can be done for early definitive diagnosis and prevention of upper urinary tract damage.

Keywords: Ano-rectal malformation, lower urinary tract dysfunction, neurovesical dysfunction, uroflowmetry.

\section{Introduction}

The patients of Ano-rectal Malformation (ARM) have high incidence of urogenital abnormalities. Upto $50-60 \%$ incidence is reported for high/intermediate ARM and 15$20 \%$ incidence for Low ARM. The incidence of lower urinary tract dysfunction (LUTD) is high in ARM, as these patients have associated spinal abnormalities (1-3). LUTD is defined as any functional anomaly of the bladder and/or urethra that has negative influence on voiding function. In patients with ARM, voiding dysfunction usually is neuropathic in origin and is commonly caused by associated defects of the lumbosacral spinal column (e.g. sacral agenesis) or abnormalities in the spinal cord (e.g. tethered spinal cord). Less commonly, iatrogenic pelvic nerve damage acquired during reconstruction of the ARM causes voiding dysfunction but

Correspondence : Dr. Rahul Saxena, Department of Pediatric Surgery, All India Institute of Medical Sciences, Jodhpur - 342005, India. Email : drrahulsaxena@gmail.com. Mobile : 9587326200. 
posterior sagittal anorectoplasty (PSARP) causes minimal injury to nerve supply of genitourinary system as there is limited rectovesical dissection (3-5). Urological abnormalities can result in severe deterioration of the upper urinary tract when treated inadequately (6) or there is delay in identification of LUTD $(7,8)$. LUTD are more common and severe in complex ARM but less complex ARM are also not free from risk to develop LUTD and therefore urodynamic study is recommended to diagnose LUTD in patients of ARM (9). The maximum flow rate and the type of flow curve obtained on noninvasive uroflowmetry may guide us for the requirement of more invasive urodynamic testing (10). With this premise in mind, the role of early uroflowmetry in anticipating the LUTD and future deterioration of upper urinary tract functioning/damage has been investigated in the present study.

\section{Material and Methods}

This prospective study conducted at a tertiary health care centre during the period from February 2012 to January 2013 which included 20 patients of ARM. The patients who had urinary tract infection (UTI) were treated before including into the study. This study aimed to determine the role of uroflowmetry in early diagnosis of LUTD in patients of ARM. The parents of patients were asked about the presence of any of the lower urinary tract symptoms (LUTS) like crying while urination, urinary hesitancy, and straining weak stream, intermittency, frequency, urgency and incontinence, wherever applicable as per age of patient and were investigated thoroughly. Hemogram, renal function test, X-ray spine, ultrasound abdomen, micturating cystourethrogram (MCU) and uroflowmetry were done in every patient atleast 6 weeks after definitive procedure. Uroflowmetry was done using Wireless Portable Urodynamic System, model Delphis. Software used is Delphis basic Urodynamic Software to display curve nomogram by Hospimedica International Ltd.
Uroflowmetry was done after ensuring good hydration and repeated in same sitting if reasonable volume was not expelled. The maximum flow rate (Qmax) was considered to be normal when the square of it was equal or more than voided volume. In addition, the maximum flow rate was considered normal only when it persisted for at least 2 seconds; other sharp peaks of shorter duration were considered artefacts. Bell shape curve of uroflowmetry was considered normal.

Twenty patients with the mean age of $3.015 \pm 0.86$ years and suffering with ARM were recruited. Out of these $12(60 \%)$ were male and $8(40 \%)$ female; 11 (55\%) were of high ARM, 4 (20\%) intermediate and $5(25 \%)$ were of low ARM.

The document associated congenital anomalies noted at birth were present in 10 $(50 \%)$ patients. Incidence of congenital anomalies in high ARM was 64\% (7/11), in Intermediate ARM was 50\% (2/4), and in Low ARM it was 25\% (1/5). Vertebral anomalies was present in 7/20 (35\%) and all had partial sacral agenesis. Urogenital anomalies were present in $6 / 20(30 \%)$ patients $(\mathrm{M}: \mathrm{F}=1: 5)$. One male child had right undescended testis. Solitary kidney was present in one, hydronephrosis (HDN) in 3 and vesico-ureteric reflux in 1 patient. CVS anomalies were present in 2/20 (10\%) patients and both had VSD. One patient $(5 \%)$ had esophageal atresia with tracheo-esophageal fistula (Table 1).

\section{Statistical Analysis}

The continuous variables were described by mean and standard deviation. Number and/or percentage was described as categorical variables. Statistical analysis was conducted by using Statistical Package for social sciences (SPSS 21, IBM, SPSS Inc., Chicago, IL, USA). The $\mathrm{P}$ value $<0.05$ was considered as significant. Categorical variables were compared by Chisquare test. 
Table 1: Patient characteristics

\begin{tabular}{|c|l|}
\hline \multicolumn{1}{|c|}{ Patient Characteristics } & $\mathbf{n = 2 0}(\%)$ \\
\hline Sex & \\
\hline Male & $12 / 20(60 \%)$ \\
\hline Female & $8 / 20(40 \%)$ \\
\hline Type of ARM & \\
\hline High & $11 / 20(55 \%)$ \\
\hline Intermediate & $4 / 20(20 \%)$ \\
\hline Low & $5 / 20(25 \%)$ \\
\hline Associated Anomalies & $10 / 20(50 \%)$ \\
\hline High ARM & $7 / 11(64 \%)$ \\
\hline Intermediate & $2 / 4(50 \%)$ \\
\hline Low & $1 / 5(20 \%)$ \\
\hline Vertebral Anomalies & $7 / 20(35 \%)$ \\
\hline High ARM & $6 / 11(55 \%)$ \\
\hline Intermediate & $1 / 4(25 \%)$ \\
\hline Low & 0 \\
\hline Urogenital Anomalies & $6 / 20(30 \%)$ \\
\hline Right UDT & $1(5 \%)$ \\
\hline Hydronephrosis (R:L) & $3(1: 2)(15 \%)$ \\
\hline VUR & $1(5 \%)$ \\
\hline Single Kidney & $1(5 \%)$ \\
\hline Gastrointestinal (EA+ TEF) & $1(5 \%)$ \\
\hline Cardiovascular (VSD) & $2(10 \%)$ \\
\hline ARM And & \\
\hline
\end{tabular}

ARM- Anorectal malformation, UDT- Undescended testis,

EA+TEF- Esophageal atresia + tracheo-esophageal fistula, VSD- Ventricular-septal defect

\section{Results}

LUTS were present in $8 / 20(40 \%)$ cases of which straining was most common followed by weak stream and crying while micturition. Straining was present in $8(40 \%)$ patients, crying and weak stream in $7(35 \%)$ each and recurrent UTI in $3(15 \%)$ patients. The mean Qmax was $7.18 \pm 3.95 \mathrm{ml} / \mathrm{sec}$ and the mean voided volume was $67.85 \pm 46.88 \mathrm{ml}$. The maximum flow was normal in $9(45 \%)$ patients and abnormal in 11 $(55 \%)$ patients. The mean voiding time was $10.45 \pm 3.08 \mathrm{~s}$ and mean time to maximum flow was $2.55 \pm 0.75 \mathrm{~s}$. The curve was Bell shape in 9 (45\%) patients and abnormal in 11 (55\%) patients. The pattern was staccato in 7 patients, interrupted in 1, plateau in 3 patients. Thus, the uroflowmetric abnormalities were present in $11 / 20(55 \%)$ patients.
LUTS was present in $8 / 20(40 \%)$ patients but uroflowmetric abnormalities were present in $11 / 20(55 \%)$ patients. Forty five percent $(5 / 11)$ of patients with abnormal uroflowmetry were asymptomatic and 25\% (2/8) symptomatic patients had normal uroflowmetry.

On follow-up Ultra-sonograph (USG), $11 / 20(55 \%)$ patients had significant post-void residual urine (PVRU) volume; 5/5 asymptomatic patients with uroflowmetry abnormalities had significant PVRU: 3 of them had previously known co-existent HDN and 1 had bladder wall thickening (BWT). Patients with HDN were evaluated to assess the renal function. Among those six symptomatic patients who had abnormal uroflowmetry, 5 (83\%) had significant PVRU; one had co-existent hydrourteronephrosis (HDUN) that was non- 
refluxing on MCU and other had BWT with HDUN who demonstrated left side vesicoureteric reflux (VUR) on MCU (Table 2).

One patient who was asymptomatic and not having uroflowmetric abnormality had significant PVRU on USG, 2 symptomatic and six asymptomatic patients who had normal uroflowmetry had no abnormality in USG.

The incidence of uroflowmetric abnormalities was significantly higher in patients with spinal anomalies $(\mathrm{p}=0.03 ; \chi 2=4.1)$. Out of twenty, seven patients had spinal anomalies and out of them $6(84 \%)$ had uroflowmetric abnormalities. Out of 13 patients with normal x-ray spine, 5(38.5\%) had uroflowmetric abnormalities. Similarly, the incidence of uroflowmetric abnormalities was significantly higher in patients with high ARM $(p=0.004 ; \chi 2=8.1)$. Uroflow metric abnormalities were present in nine out of 11 patients $(82 \%)$ with high ARM, 2/4 (50\%) of intermediate ARM and none who had low ARM (Fig. 1).

\section{Discussion}

The association of neurovesical dysfunction (NVD) with ARM is commonly neurogenic and caused by neuropathological disorders associated with sacral and spinal abnormalities $(1,4)$ and less commonly due to iatrogenic pelvic nerve damage $(3,8)$. The LUTS are categorically classified according to their relation to storage or voiding phase of bladder function. The storage symptoms are increased or decreased frequency, incontinence, urgency and nocturia, while voiding symptoms are hesitancy, straining, weak stream and intermittency (10). Other symptoms include holding manoeuvres like standing on tiptoe, forcefully crossing the legs or squatting with the heel pressed into the perineum (11), feeling of incomplete emptying, post-void dribbling and genital or lower urinary tract pain. Most of these

\section{Table 2: Ultrasound findings of patients with uroflowmetric abnormalities}

\begin{tabular}{|c|c|}
\hline Associated anomaly & Follow up USG \\
\hline 1. Single Kidney & PVRU \\
\hline 1. $\quad$ RHDN & RHDN/PVRU \\
\hline 1. $\quad$ LDHN & LHDN/PVRU \\
\hline 1. LHDN & LHDN/PVRU \\
\hline 1. None & BWT/PVRU \\
\hline \multicolumn{2}{|c|}{ Symptomatic patients with Uroflowmetric abnormalities } \\
\hline Associated anomaly & Follow up USG \\
\hline 1. $\quad$ Right UDT & PVRU \\
\hline 1. EA+TEF & PVRU \\
\hline 1. Cardiac anomaly -VSD & PVRU \\
\hline 1. Left VUR & BWT+HDUN+PVRU \\
\hline 1. None & HDUN+PVRU \\
\hline 1. None & NAD \\
\hline
\end{tabular}

PVRU-Post void residual volume, RHDN-right hydronephrosis, LHDN-Left hydronephrosis,

BWT-Bladder wall thickening, UDT-Undescended testis, HDUNhydroureteronephrosis, NAD-No abnormality detected 


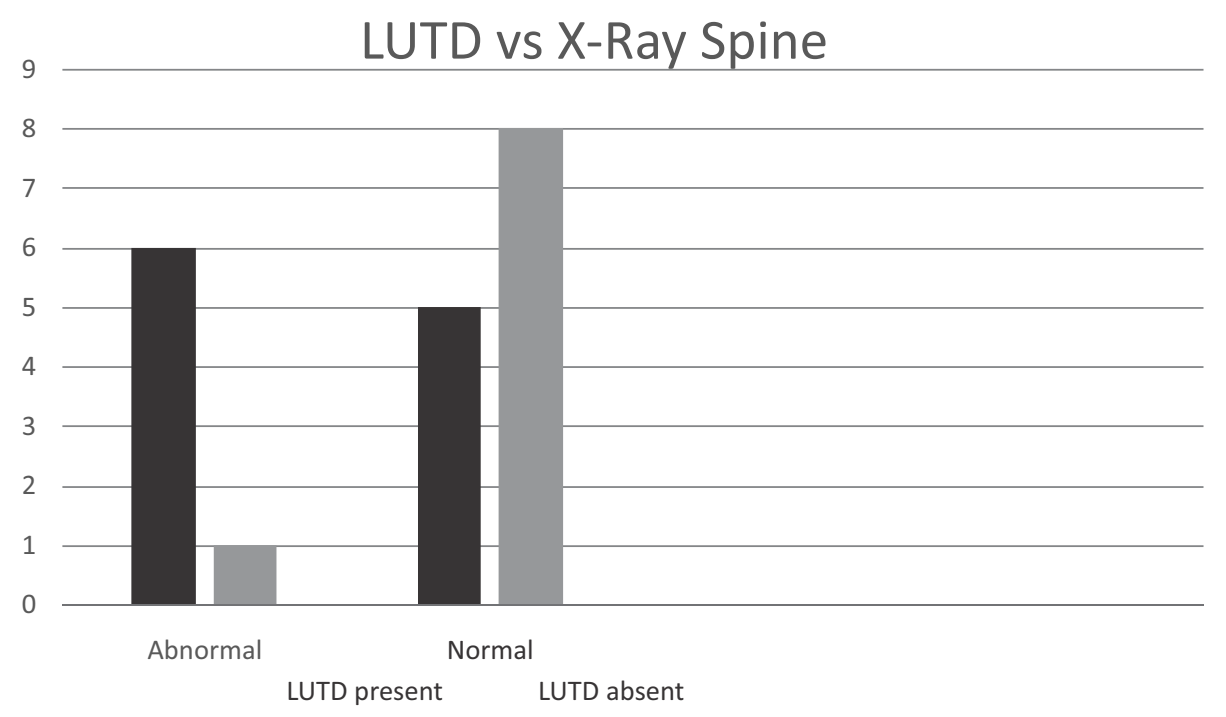

Fig. 1: Correlation of lower urinary tract dysfunction in patients with abnormalities in $\mathrm{X}$-ray spine

symptoms, can be appreciated after age of 5 years, except straining which is applicable in all ages, weak stream that is relevant from infancy and intermittency which is considered physiological until 3 years of age (10). In our study since most of the patients were less than 5year old, symptoms which were focussed upon were straining, weak stream and crying while micturition.

The PVRU is considered significant when it is more than acceptable limit of $10 \%$ of bladder capacity in adults but it is not relevant in infants and children. A range of 5 to $20 \mathrm{ml}$ may be associated with insufficient emptying, so that the examination should be repeated. More than 20 $\mathrm{ml}$ residual urine found on repetitive occasions indicates abnormal or incomplete emptying, if time gap between urination and ultrasound should not be more than 5 minutes and child should not have waited over ambitiously for urination so that he has achieved a state of bladder fullness in excess of what is normal for the patient (10). In our study, PVRU volume was high in eleven patients.

Measurement of urine flow and residual urine (with ultrasound) as a stand-alone examination is by far the most common procedure in paediatric urodynamic practice. To a large degree, the results of the flow/residual examination decide whether the child requires an invasive urodynamic investigation. The Qmax is most important parameter when assessing bladder outflow and is considered normal when the square of it was equal or more than voided volume (10). There is linear correlation of square of maximum flow and voided volume in normal individuals (12).

In our study, 5/11 patients with uroflowmetric abnormality were asymptomatic but all of them showed radiologic abnormality suggestive of bladder outlet obstruction. The $2 / 8$ patients with LUTS did not show any uroflowmetric abnormality but none of these patients were found to have any radiologic finding suggestive of bladder outlet obstruction. These findings suggest that uroflowmetry is a more sensitive investigation to detect functional or anatomical bladder outlet obstruction than LUTS alone. Being a non-invasive investigation, it can be used as a screening test to identify the patients who require urodynamic study.

Jindal et al (8) reported 70\% incidence of neurovesical dysfunction in ARM and in our 
series, uroflowmetric abnormalities were present in 11 (55\%) cases. Emir and Soylet (13) had reported a $45.4 \%$ incidence of neurovesical dysfunction, 70\% with supra-levator anal atresia and $34.7 \%$ in patients with infra-levator anal atresia. Mosiello et al (14) had reported, overall a $35 \%$ incidence of neurovesical dysfunction, $51 \%$ with high ARM and 40\% with low ARM and Kakizaki et al (1) reported a 100\% incidence of NVD in patients with high ARM. We have also observed that uroflowmetric abnormality was present in significantly higher number of patients with supra-levator ARM with presence of uroflowmetric abnormality in $82 \%$ of High ARM and $50 \%$ of Intermediate ARM.

Goosens et al (9) reported 43\% incidence of LUTD in ARM with vertebral anomalies and $8 \%$ with no vertebral anomalies and in present study $6 / 7(84 \%)$ of patients with vertebral anomalies had LUTD but it was also present in 5/13 (38.5\%) patients with normal X-ray spine.

Borg et al (15) studied LUTD longitudinally and assessed the bladder function at ages 5, 10 and 15 years. The LUTD was classified as neurogenic and non-neurogenic and they found that dysfunction in neurogenic group was permanent while non-neurogenic LUTD was often transient and mild. The number of children with non-neurogenic LUTD was higher in the lower age group at 5 years. The only predictor of LUTD overall (neurogenic and nonneurogenic LUTD together) was spinal cord malformation at both 5-year and 10-year followup. In our study, we also found that uroflowetric abnormalities are significantly higher in patients with vertebral anomalies.

\section{Limitations of Study}

In our study due to restricted duration, the sample size is small. MRI spine was not done in patients with NVD and cystometry and specific management strategy were not included in study.

\section{Conclusion}

Uroflowmetric abnormalities are present in significantly higher number of patients with vertebral anomalies; however normal X-ray spine does not rule out NVD and also their incidence is significantly higher in high ARM. All patients after PSARP should be followed clinically and radiologically for protection of upper urinary tract damage. Uroflowmetry is a non-invasive method that may help in early detection of NVD in asymptomatic patients and subsequently cystometric analysis can be performed earlier in the patients with uroflowmetric abnormalities that may help in ascertaining with early definitive diagnosis and planning of management strategy to prevent upper urinary tract damage.

\section{Conflict of Interest}

None

\section{References}

1. Kakizaki H, Nanomura K, Asano Y, et al (1994). Preexisting neurogenic voiding dysfunction in children with imperforate anus: problems in management. $J$ Urol 151:1041-1044.

2. Sheldon C, Cormier M, Crone K, et al (1991). Occult neurovesical dysfunction in children with imperforate anus and its variants. J Pediatr Surg 26: 49-54.

3. Boemers TM, Bax KM, Rovekamp MH, et al (1995). The effect of posterior saggital anorectoplasty and its variant on lower urinary tract function in children with anorectal malformations. J Urol 153:19193.

4. Boemers TM, Beek FJ, van Gool JD, de Jong TP, Bax KM (1996). Urologic problems in anorectal malformation. Part 1: urodynamic findings and significance of sacral anomalies. J Pediatr Surg 31:407410. 
5. Pena A, Devries PA (1982). Posterior saggital anorectoplasty: important technical considerations and new applications. JPediatr Surg 17:796-811.

6. Boemers TM, de Jong TP, van Gool JD, Bax KM (1996). Urologic problems in anorectal malformations. Part 2: functional urologic sequelae. J Pediatr Surg 31(5): 634-637.

7. Boemers TM, Beek FJ, Bax NM (1999). Guidelines for the urological screening and initial management of lower urinary tract dysfunction in children with anorectal malformations - the ARGUS protocol. BrJ Urol Int 83(6): 662-671.

8. Jindal B, Grover VP, Bhatnagar V (2009). The assessment of lower urinary tract function in children with anorectal malformations before and after PSARP. Eur J Pediatr Surg 19(1): 34-37.

9. Goossens WJ, de Blaauw I, Wijnen $\mathrm{MH}$, de Gier RP, Kortmann B, Feitz WF (2011). Urological anomalies in anorectal malformations in The Netherlands: effects of screening all patients on long-term outcome. Pediatr Surg Int 27: 1091-1097.
10. Neveus T, von Gontard A, Hoebeke P, et al (2006). The standardization of terminology of lower urinary tract function in children and adolescents: report from the Standardisation Committee of the International Children's Continence Society. JUrol 176: 314-324.

11. Vincent SA (1996). Postural control of urinary incontinence. The curtsy sign. Lancet 2: 631-632.

12. Szabo L, Fegyverneki S (1995). Maximum and average urine flow rates in normal children - the Miskolc nomograms. Br J Urol Int 76: 16-20.

13. Emir H, Soylet Y (1998). Neurovesical dysfunction in patients with anorectal malformations. Eur J Pediatr Surg 8:9597.

14. Mosiello G, Capitanucci ML, Gatti C, et al (2003). How to investigate neurovesical dysfunction in children with anorectal malformation. JUrol 170:1610-1613.

15. Borg H, Holmdahl G, Doroszkievicz M, Sillen U (2014). Longitudinal study of lower urinary tract function in children with anorectal malformation. Eur $J$ Pediatr Surg 24:492-499. 